\title{
Posttraumatic Epidural Haematoma in a child with severe haemophilia A - case study
}

\author{
Grzegorz Starobrat ${ }^{1}$, Jolanta Taczała ${ }^{2}$, Marek Fatyga', Katarzyna Baran ${ }^{3}$, Kacper Sowa', $^{1}$ \\ Monika Sarna', Magdalena Maria Woźniak ${ }^{3}$, Michał Latalski' ${ }^{1}$, Anna Danielewicz ${ }^{1}$ \\ ${ }^{1}$ Department of Pediatric Orthopaedics, Medical University of Lublin, Poland \\ ${ }^{2}$ The Department of Rehabilitation and Physiotherapy the Faculty of Health Sciences, Medical University in Lublin, Poland \\ ${ }^{3}$ Department of Pediatric Radiology, Medical University of Lublin, Poland
}

Starobrat G, Taczała J, Fatyga M, Baran K, Sowa K, Sarna M, Woźniak M. M, Latalski M, Danielewicz A. Posttraumatic Epidural Haematoma in a child with severe haemophilia A - case study. J Pre-Clin Clin Res. 2019; 13(2): 76-78. doi: 10.26444/jpccr/106100

\begin{abstract}
This paper presents the case of a 5-year-old child with severe haemophilia A treated for epidural bleeding into the CNS resulting from spine trauma. Every case of CNS trauma in children with haemophilia entails a high threat to life and limb, and as a priority requires factor VIII supplementation and further urgent diagnostics. Treating a child with CNS bleeding in the course of haemophilia requires the cooperation of a team of specialists, including a haematologist, radiologist, neurosurgeon, orthopedist and rehabilitation specialist. The treatment strategy in itself and selection of a conservative or surgical treatment method require an in-depth analysis. Supplementation treatment should be initiated immediately following the diagnosis of a head or spinal column injury, or after the onset of signs and symptoms indicating CNS bleeding.
\end{abstract}

\section{Key words}

haemophilia A, early intervention, MR imaging, spinal epidural haematoma, neurological deficits

\section{INTRODUCTION}

In children with haemophilia, regardless of the type, trauma of the central nervous system with complications in the form of bleeding, constitute very dangerous conditions that may be directly life threatening. These occur in $2-12 \%$ of children with haemophilia and mainly include bleeding into the central nervous system following head trauma, and very rarely bleeding in the spinal cord area. $90 \%$ of the spinal cord area bleeding cases are epidural and $10 \%$ of the cases are intraspinal [1-4].

The risk of bleeding itself increases with severity of the underlying disease and number of past trauma [2]. A suspected bleeding in children with haemophilia warrants initiating coagulation factor supplementation, extensive urgent radiological diagnostics and a surgical intervention in case of continued intensification of symptoms.

The purpose of this paper is to discuss the case of a child with post-traumatic epidural haematoma of the spinal canal in the course of severe haemophilia A, taking into consideration aspects of diagnostics, treatment and rehabilitation.

\section{CASE STUDY}

A 5-year-old boy with haemophilia A diagnosed at the 2nd month of life, treated at the Haematology Outpatient Clinic, suffered spinal column trauma after falling off a snow sled. Reported acute pain in the thoracic and lumbar region. There was no history of trauma, diarrhea, fever, vomiting, skin or orificial bleed and joint swelling. There was no loss of consciousness, headache and seizures. Physical examination revealed spastic paresis of the lower extremities,

Adress for correspondence: Michał Latalski, Department of Pediatric Orthopaedics, Medical University of Lublin, Poland

E-mail: michall1@02.pl

Received: 12 March 2019; accepted: 1 April 2019; first published: 12 April 2019 more pronounced on the left side; dysesthesia, especially on the right side; bilateral foot clonus and positive Babinski sign. Paresis of the extremities prevented independent movement. An urgent spinal column tomography (CT) confirmed an epidural haematoma at the Th 11-L 2 level (Fig. 1). Supplementation of recombinant factor VIII was started from the very beginning, as per applicable recommendations, at a dose of $50 \mathrm{U} / \mathrm{kg}$. The patient was treated surgically in urgent mode by a neurosurgery team. A laminectomy procedure was performed at the Th 11-L 2 level with evacuation of the haematoma and concomitant stabilization. Treatment with

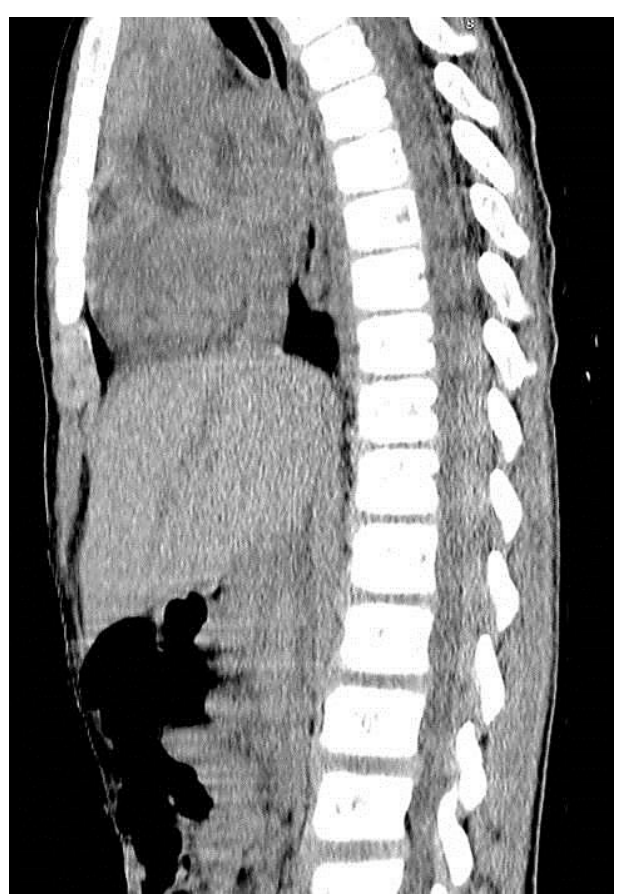

Figure 1. Spinal column tomography (CT) showing an epidural haematoma at the Th 11-L 2 level 

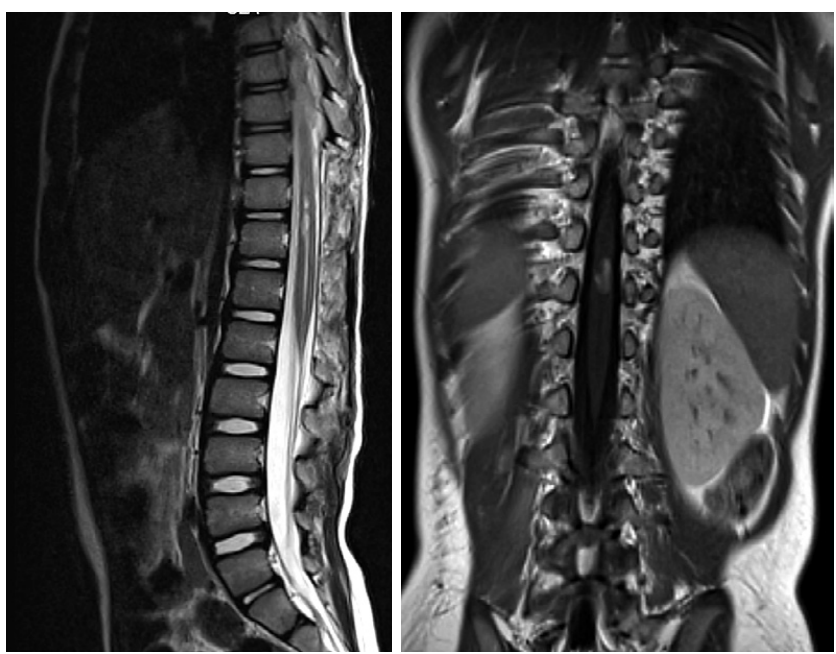

Figure 2. Magnetic resonance imaging (MRI) performed after the surgical procedure

recombinant factor VIII was continued at a dose ensuring activity at a level of 80-100 IU/dL, gradually reducing the activity to $50 \mathrm{IU} / \mathrm{dL}$ until day 14 following the procedure. Factor levels were assayed daily. A follow-up magnetic resonance imaging (MRI) performed after the surgical procedure revealed no new haemorrhagic foci in the spinal cord (Fig. 2).

Following the acute trauma period, and three weeks following the procedure, the patient underwent comprehensive rehabilitation at the Neuro-rehabilitation Department, initially at the bedside, involving passive and active exercises. A spinal jacket was made and adjusted to the patient, securing the spine against secondary kyphotic curve. Passive vertical positioning on a tilting table was performed at the Neurorehabilitation Department, followed after a week by active vertical positioning, and then teaching him to walk independently in the spinal jacket, six weeks after the procedure. For the entire rehabilitation period, the child received rehabilitation factor VIII to maintain his activity at the required level.

Upon discharge, a physical examination revealed a significant increase in the lower extremities muscle strength (4-5 on the Lovett scale), improvement in the strength of postural muscles, no dysesthesias, and resolution of foot clonus. Retained increased tension and spasticity within the triceps surae and bilateral Babinski sign.

Surgical treatment along with long-term factor VIII supplementation led to a reduction of the cord injury, which was confirmed in a follow-up MRI performed after three months. Systematic rehabilitation at the Neurorehabilitation Department, performed for a total of four months, led to an improvement in the motor function of the lower extremities, resolution of dysesthesias and spasticity. The boy recovered the ability to walk independently with a pattern similar to the correct pattern.

A follow-up examination after two years revealed incomplete knee extension while walking with a $15 \mathrm{deg}$. functional contracture. Despite wearing an orthopedic jacket, follow-up plain radiograms revealed a tendency for kyphotic deformation of the spinal axis at the height of the laminectomy (Fig. 3). The patient remains under the continuous care of an orthopedist.

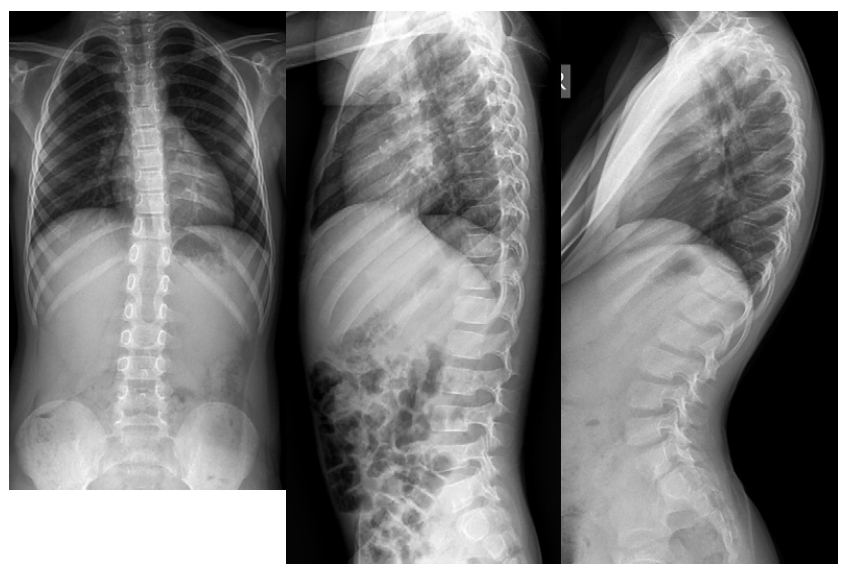

Figure 3. Radiograms revealed a tendency for kyphotic deformation of the spinal axis at the height of the laminectomy

\section{DISCUSSION}

Haemophilia is a genetic bleeding disorder inherited asa recessive train linked to the male gender. The incidence rate of haemophilia remains at the level of $0.7-0.8$ per 10,000 newborns. Haemophilia is caused by the lack or deficiency of coagulation factors: VIII for type A and IX for type B [3].

Bleeding into the central nervous system in patients with haemophilia is a very dangerous condition that may be directly life-threatening, and its complications may lead to significant neurological deficits. It is estimated that bleeding into the CNS occur in $2-12 \%$ of children with haemophilia $[1,4]$. Bleeding cases have been reported in children below one year of age [5]. Bleeding into the spinal cord occurs rarely and usually takes an acute course, although reports of cases with a chronic course have been described in literature [6].

In the case of CNS trauma or suspected trauma in a haemophilia patient, supplementation treatment should be introduced immediately before performing imaging diagnostics [7]. Coagulation factor activity level should be assayed separately. In the case of haemophilia A, the dose is selected so that the first seven days allow achievement of an activity level of $80-100 \mathrm{IU} / \mathrm{dL}$ as the starting dose, followed by a maintenance dose of 50 IU/dL over the next 14 days [8].

Magnetic resonance imaging should be a diagnostic modality of choice for radiological diagnostics of patients with haemophilia, as it allows to detect even a minor bleeding focus [6]. Due to the limited availability of magnetic resonance, many centres perform computed tomography as the first modality.

Treatment of children with spinal cord haematoma in the course of haemophilia consists mainly in the substitution of the deficient factor, as described above. Nirupam et al. report that the surgical decision should be taken following an assessment of the patient's condition, intensification of neurological signs and symptoms and intensification of bleeding identified in MRI or CT [9]. Similarly, Kubota and Miyajima believe that supplementation with high doses of recombinant factor may allow treatment and lead to the resolution of the neurological signs of an epidural hematoma without the need for surgical intervention in patients with bleeding disorders [10]. However, the patients they described presented with moderate clinical signs. In the case of major neurological deficits related to local spinal cord compression, 
urgent surgical intervention with simultaneous factor supplementation warrants consideration, and have led to swift clinical improvement in the presented case.

Where a major laminectomy is performed, one has to take into consideration the possibility of increasing spine kyphotization, especially in patients with immature skeletal systems.

\section{CONCLUSIONS}

1) Trauma to the central nervous system in haemophilia patients should be treated as potentially life-threatening conditions.

2) Supplementation treatment should be initiated immediately following the diagnosis of a head or spinal column injury, or after the onset of signs and symptoms indicating CNS bleeding.

3) Imaging and laboratory diagnostics are urgent.

4) Surgical evacuation of the CNS is a treatment option for Post-traumatic Epidural Haematoma.

\section{REFERENCES}

1. Kiehna EN, Waldron PE, Jane JA. Conservative management of an acute spontaneous holocord epidural hemorrhage in a haemophiliac infant. J Neurosurg Pediatr. 2010; 6: 43-8.

2. Ljung RCR. Intracranial haemorrhage in haemophilia A and B. Br J Haematol. 2007; 140: 378-384.

3. Agrawal D, Mahapatra AK. Spontaneous subdural hematoma in a young adult with haemophilia. Neurol India. 2003; 51: 114-5.

4. Nelson MD, Maeder MA, Usner D, et al. Prevalence and incidence of intracranial haemorrhage in a population of children with haemophilia. Haemophilia 1999; 5: 306-312.

5. Noth I, Hutter JJ, Meltzer PS, Damiano ML, Carter LP. Spinal epidural hematoma in the hemophilic infant. Am J Pediatr Hematol Oncol. 1993; 15: $131-4$.

6. Stanley P, McComb JG. Chronic spinal epidural hematoma in Haemophilia A in a child. Pediatr Radiol. 1983; 13: 241-3.

7. Windyga J, Chojnowski K, Klukowska A, et al. Wytyczne postępowania whemofilii A i B niepowikłanej inhibitorem czynnika VIII i IX (wydanie zaktualizowane). Acta Haematologica Polonica 2016; 47(2): 86-114.

8. Hoots WK. Emergency care issues in haemophilia. World Federation of Haemophilia 2007;

9. Indian J Hematol Blood Transfus. 2014 Sep; 30(Suppl 1): 54-56. Published online 2013 Mar 7. doi: 10.1007/s12288-013-0245-4

10. Kubota T, Miyajima Y. Spinal extradural haematoma due to haemophilia A. Arch Dis Child. 2007; 92: 498. 